\title{
Oscillation Criteria of Certain Third-Order Differential Equation with Piecewise Constant Argument
}

\author{
Haihua Liang and Gen-qiang Wang \\ Department of Computer Science, Guangdong Polytechnic Normal University, Guangzhou 510665, \\ Guangdong, China \\ Correspondence should be addressed to Haihua Liang, haiihuaa@tom.com
}

Received 17 August 2012; Revised 27 October 2012; Accepted 20 November 2012

Academic Editor: Samir H. Saker

Copyright (C) 2012 H. Liang and G.-q. Wang. This is an open access article distributed under the Creative Commons Attribution License, which permits unrestricted use, distribution, and reproduction in any medium, provided the original work is properly cited.

We study the oscillation and asymptotic behavior of third-order nonlinear delay differential equation with piecewise constant argument of the form $\left(r_{2}(t)\left(r_{1}(t) x^{\prime}(t)\right)^{\prime}\right)^{\prime}+p(t) x^{\prime}(t)+f(t, x([t]))=$ 0 . We establish several sufficient conditions which insure that any solution of this equation oscillates or converges to zero. Some examples are given to illustrate the importance of our results.

\section{Introduction}

Let $[\cdot]$ denote the greatest-integer function. Consider the following third-order nonlinear delay differential equation with piecewise constant argument:

$$
\left(r_{2}(t)\left(r_{1}(t) x^{\prime}(t)\right) \prime\right)^{\prime}+p(t) x^{\prime}(t)+f(t, x([t]))=0, \quad t \geq 0,
$$

where $r_{1}(t), r_{2}(t)$ are continuous on $[0, \infty)$ with $r_{1}(t), r_{2}(t)>0, p(t)$ is continuously differentiable on $[0, \infty)$ with $p(t) \geq 0$. We will show that every solution $x(t)$ of $(1.1)$ oscillates or converges to zero, provided appropriate conditions are imposed.

Throughout this paper, we assume that $x f(t, x) \geq 0$ and that there exist functions $q(t)$ and $\phi(x)$ such that

(i) $q(t)$ is continuous on $[0, \infty)$ with $q(t)>0$,

(ii) $\phi(x)$ is continuously differentiable and nondecreasing on $(-\infty,+\infty), \phi(x) / x \geq K>$ 0 for $x \neq 0$, 
(iii) $|f(t, x)| \geq q(t)|\phi(x)|, x \neq 0, t \geq 0$,

(iv) $K q(t)-p^{\prime}(t) \geq 0$ and is not identically zero in any subinterval of $[0, \infty)$.

The delay functional differential equations provide a mathematical model for a physical or biological system in which the rate of change of the system depends upon its past history. In recent years, the oscillation theory and asymptotic behavior of delay functional differential equations and their applications have been and still are receiving intensive attention. In fact, in the last few years several monographs and hundreds of research papers have been written, see, for example [1-4]. In particular case, determining oscillation criteria for second-order delay differential equations has received a great deal of attention, while the study of oscillation and asymptotic behavior of the third-order delay differential equations has received considerably less attention in the literature.

The delay differential equations with piecewise continuous arguments can be looked as a special kind of delay functional differential equations. Since the delays of such equations are discontinuous, it need to be investigated individually. As is shown in [5], the solutions of differential equations with piecewise continuous arguments are determined by a finite set of initial data, rather than by an initial function as in the case of general functional differential equations. Moreover, the strong interest in such equations is motivated by the fact that they represent a hybrid of continuous and discrete dynamical systems and combine the properties of both differential and difference equation.

In the last few decades, there has been increasing interest in obtaining sufficient conditions for the oscillatory of solutions of different classes of the first-order differential equations with piecewise constant arguments, see [6-9] and the references therein. It is found that the presence of piecewise constant arguments plays an important role in the oscillation of the solution. For instant, all solution of $x^{\prime}+2 x([t])=0$ are oscillatory (see [6]). But the corresponding ordinary differential equation $x^{\prime}+2 x=0$ has nonoscillatory solution $x=e^{-2 t}$.

However, there are few results about the oscillation of higher order equations. As mentioned in $[5,10]$, there are reasons for investigating the higher order equations with piecewise constant arguments. For example, suppose a moving particle with time variable mass $r(t)$ is subjected to a restoring controller $-\phi(x([t]))$ which acts at sampled time $[t]$, then the second law of motion asserts that

$$
\left(r(t) x^{\prime}(t)\right)^{\prime}+\phi(x([t]))=0 .
$$

In [10], the authors study a slightly more general second-order delay differential equations of the form:

$$
\left(r(t) x^{\prime}(t)\right)^{\prime}+f(t, x([t]))=0, \quad t \geq 0
$$

Two sufficient conditions which insure that any solution of (1.3) oscillates are obtained.

However, as far as we know, there are not works studying the oscillation and asymptotic behavior of third-order delay differential equations with piecewise constant argument. Motivated by this fact, in the present paper, we will investigate the oscillatory and asymptotic behavior of a certain class of third-order equation (1.1) with damping. The main ideas we used here are based on the paper $[3,4,10]$.

The rest of this paper is organized as follows. In Section 2 we give the definition of the solution of (1.1) and establish some lemmas which are useful in the proof of our main results. 
Section 3 is devoted to the presentation of several sufficient conditions for the oscillation of (1.1). In Section 4, several examples are given to illustrate the importance of our results.

\section{Definitions and Preliminary Lemmas}

Similar to [11], we give the following definition.

Definition 2.1. A solution of $(1.1)$ on $[0, \infty)$ is a function $x$ that satisfies the conditions:

(i) $\left(r_{1}(t) x^{\prime}(t)\right)^{\prime}$ is continuous on $[0, \infty)$,

(ii) $r_{2}(t)\left(r_{1}(t) x^{\prime}(t)\right)^{\prime}$ is differentiable at each point $t \in[0, \infty)$, with the possible exception of the points $[t] \in[0, \infty)$, where one-sided derivatives exist,

(iii) Equation (1.1) is satisfied on each interval $[k, k+1) \subset[0, \infty)$ with $k \in \mathbf{N}$.

Our attention is restricted to those solutions of (1.1) which exist on the half line $[0, \infty)$ and satisfy $\sup \{|x(t)|: T \leq t<\infty\}>0$ for any $T>0$. We make a standing hypothesis that (1.1) does possess such solutions. As usual, a solution of (1.1) is called oscillatory if it has arbitrarily large zeros and nonoscillatory otherwise. Equation (1.1) itself is called oscillatory if all its solutions are oscillatory.

Remark 2.2. If $x$ is a solution of (1.1), then $y=-x$ is a solution of the equation

$$
\left(r_{2}(t)\left(r_{1}(t) y^{\prime}(t)\right)^{\prime}\right)^{\prime}+p(t) y^{\prime}(t)+\tilde{f}(t, y([t]))=0, \quad t \geq 0
$$

where $\tilde{f}(t, y)=-f(t,-y)$. It is easy to check that $y \tilde{f}(t, y)>0$ and $|\tilde{f}(t, y)| \geq q(t)|\tilde{\phi}(y)|$, where $\tilde{\phi}(y)=-\phi(-y)$ with $\tilde{\phi}(y) / y \geq K$. Thus, concerning nonoscillatory solutions of (1.1), we can restrict our attention only to the positive ones.

For the sake of brevity, we denote the following two operators

$$
L_{1} x(t)=r_{1}(t) x^{\prime}(t), \quad L_{2} x(t)=r_{2}(t)\left(L_{1} x(t)\right)^{\prime} .
$$

Thus (1.1) can be written as

$$
\left(L_{2} x(t)\right)^{\prime}+p(t) x^{\prime}(t)+f(t, x([t]))=0, \quad t \geq 0 .
$$

Similar to [12], we give the following definition.

Definition 2.3. Let $x$ be a solution of (1.1). We say that $x$ has property $V_{2}$ on $[T, \infty), T \geq 0$, if $x(t) L_{k} x(t)>0, i=1,2$ for every $t \in[T, \infty)$.

It is worth pointing out here that if $x$ has property $V_{2}$ on $[T, \infty)$, then by $(1.1)$, $x(t)\left(L_{2} x(t)\right)^{\prime}$ is eventually nonpositive.

Define the functions

$$
R_{i}(t, T)=\int_{T}^{t} \frac{d s}{r_{i}(s)}, \quad i=1,2
$$


We assume that

$$
\begin{aligned}
& R_{1}(t, T) \longrightarrow \infty, \quad \text { as } t \longrightarrow \infty, \\
& R_{2}(t, T) \longrightarrow \infty, \quad \text { as } t \longrightarrow \infty
\end{aligned}
$$

To obtain our main results we need the following lemmas.

Lemma 2.4. Suppose that

$$
r_{1}(t)\left(r_{2}(t) y^{\prime}(t)\right)^{\prime}+p(t) y(t)=0
$$

is nonoscillatory. If $x$ is a nonoscillatory solution of (1.1), then $x \prime(t)$ does not change sign eventually.

Proof. Suppose that $x(t)$ is a nonoscillatory solution of $(1.1)$ on $[0, \infty)$. Without loss of generality, we may assume that $x(t)>0$ for $t \geq 0$. Let $y(t)$ be a nonoscillatory solution of (2.7). We will firstly consider the case that $y(t)$ is eventually negative, that is, there exists a constant $T_{0}$ such that $y(t)<0$ for $t \geq T_{0}$. By (1.1) and (2.7), it is easy to see that

$$
\begin{aligned}
& \left(r_{2}(t)\left(r_{1}(t) x^{\prime}(t)\right)^{\prime} y(t)-r_{2}(t) y^{\prime}(t) r_{1}(t) x^{\prime}(t)\right)^{\prime} \\
& \quad=\left(r_{2}(t)\left(r_{1}(t) x^{\prime}(t)\right)^{\prime}\right)^{\prime} y(t)-\left(r_{2}(t) y^{\prime}(t)\right)^{\prime} r_{1}(t) x^{\prime}(t) \\
& =-y(t) f(t, x(k)) \geq-y(t) q(t) \phi(x(k)), \quad t \in[k, k+1), k \in \mathbf{N}, k \geq T_{0} .
\end{aligned}
$$

Suppose to the contrary that $x^{\prime}(t)$ has arbitrarily large zeros, then there exist consecutive zeros of $x^{\prime}(t), t_{1}$, and $t_{2}$, such that $t_{2}>t_{1}>T_{0}$ and $x^{\prime \prime}\left(t_{1}\right) \leq 0, x^{\prime \prime}\left(t_{2}\right) \geq 0$. If there exists an integer $k_{1}$ such that

$$
k_{1}-1 \leq t_{1}<k_{1}<k_{1}+1<\cdots<k_{1}+m<t_{2} \leq k_{1}+m+1 \text {, }
$$

see Figure 1(a), then integrating (2.8) we find

$$
\begin{aligned}
r_{2}(t)\left(r_{1}(t) x^{\prime}(t)\right)^{\prime} y(t) \mid \begin{array}{l}
t=t_{2} \\
t=t_{1}
\end{array}= & \left(r_{2}(t)\left(r_{1}(t) x^{\prime}(t)\right)^{\prime} y(t)-r_{2}(t) r_{1}(t) x^{\prime}(t) y^{\prime}(t)\right) \mid \begin{array}{l}
t=t_{2} \\
t=t_{1}
\end{array} \\
= & \int_{t_{1}}^{t_{2}}\left(r_{2}(t)\left(r_{1}(t) x^{\prime}(t)\right)^{\prime} y(t)-r_{2}(t) r_{1}(t) x^{\prime}(t) y^{\prime}(t)\right)^{\prime} d t \\
= & \int_{t_{1}}^{k_{1}}\left(r_{2}(t)\left(r_{1}(t) x^{\prime}(t)\right)^{\prime} y(t)-r_{2}(t) r_{1}(t) x^{\prime}(t) y^{\prime}(t)\right)^{\prime} d t \\
& +\int_{k_{1}}^{k_{1}+1}\left(r_{2}(t)\left(r_{1}(t) x^{\prime}(t)\right)^{\prime} y(t)-r_{2}(t) r_{1}(t) x^{\prime}(t) y^{\prime}(t)\right)^{\prime} d t \\
& +\cdots+\int_{k_{1}+m}^{t_{2}}\left(r_{2}(t)\left(r_{1}(t) x^{\prime}(t)\right)^{\prime} y(t)-r_{2}(t) r_{1}(t) x^{\prime}(t) y^{\prime}(t)\right)^{\prime} d t
\end{aligned}
$$


Journal of Applied Mathematics

$$
\begin{aligned}
\geq & \phi\left(x\left(k_{1}-1\right)\right) \int_{t_{1}}^{k_{1}}|y(t)| q(t) d t+\phi\left(x\left(k_{1}\right)\right) \int_{k_{1}}^{k_{1}+1}|y(t)| q(t) d t \\
& +\cdots+\phi\left(x\left(k_{1}+m\right)\right) \int_{k_{1}+m}^{t_{2}}|y(t)| q(t) d t>0 .
\end{aligned}
$$

Note that $\left.\left(r_{1}(t) x^{\prime}(t)\right)^{\prime}\right|_{t=t_{i}}=r_{1}\left(t_{i}\right) x^{\prime \prime}\left(t_{i}\right)(i=1,2)$, it follows from $x^{\prime \prime}\left(t_{1}\right) \leq 0, x^{\prime \prime}\left(t_{2}\right) \geq 0$ that

$$
r_{2}(t)\left(r_{1}(t) x^{\prime}(t)\right)^{\prime} y(t) \mid \begin{aligned}
& t=t_{2} \\
& t=t_{1}
\end{aligned} \leq 0
$$

which is contrary to (2.10).

If there exists an integer $k_{0}$ such that $k_{0} \leq t_{1}<t_{2} \leq k_{0}+1$, see Figure $1(\mathrm{~b})$, then integrating (2.8) directly from $t_{1}$ to $t_{2}$, we also get a contradiction.

Next we consider the case that $y(t)$ is eventually positive. Let $T_{1}$ be the constant such that $y(t)>0$ for $t \geq T_{1}$. It follows from (1.1) and (2.7) that,

$$
\left(r_{2}(t)\left(r_{1}(t) x^{\prime}(t)\right)^{\prime} y(t)-r_{2}(t) y^{\prime}(t) r_{1}(t) x^{\prime}(t)\right)^{\prime} \leq-y(t) q(t) \phi(x(k)),
$$

where $t \in[k, k+1)$ with $k \in \mathbf{N}$ and $k \geq T_{1}$. Suppose to the contrary that $x^{\prime}(t)$ has arbitrarily large zeros, then there exist consecutive zeros of $x^{\prime}(t), t_{3}$, and $t_{4}$, such that $t_{4}>t_{3}>T_{1}$ and $x^{\prime \prime}\left(t_{3}\right) \geq 0, x^{\prime \prime}\left(t_{4}\right) \leq 0$. Using the argument as above, we also arrive at a contradiction. Thus the proof is complete.

Remark 2.5. Lemma 2.4 shows that, the oscillatory or asymptotic behavior of (1.1) is linked to nonoscillation of the second-order homogeneous equation (2.7). The source of this interesting phenomenon can be explained as follows.

In (1.1), if we let $y=r_{1} x^{\prime}$, then $y$ verifies the following equation:

$$
r_{1}(t)\left(r_{2}(t) y^{\prime}(t)\right)^{\prime}+p(t) y(t)+r_{1}(t) f(t, x([t]))=0
$$

Let $x(t)$ be any solution of (1.1). If $x(t)$ is nonoscillatory, then $r_{1}(t) f(t, x([t]))$ and thus $r_{1}(t)\left(r_{2}(t) y^{\prime}(t)\right)^{\prime}+p(t) y(t)$ is eventually of one sign (see (2.13)). Hence, by the comparison method (as was shown by the proof of Lemma 2.4), the assumption that (2.7) is nonoscillatory guarantees that

$$
r_{1}(t)\left(r_{2}(t) y^{\prime}(t)\right)^{\prime}+p(t) y(t)<0 \quad \text { or } \quad(>0)
$$

is nonoscillatory. This fact means that, under the hypotheses of Lemma 2.4, any solution $x(t)$ of (1.1) is either oscillatory or is monotone.

Lemma 2.6. Suppose that assumption (2.6) is satisfied and that $x$ is a nonoscillatory solution of (1.1) such that $x(t) L_{1} x(t)>0$ for every $t \geq T \geq 0$. Then $x$ has property $V_{2}$ on $\left[T_{1}, \infty\right)$ for some $T_{1}$. 


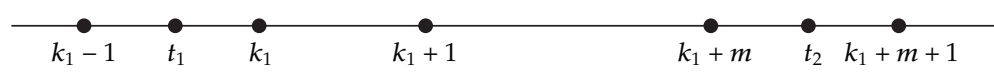

(a)

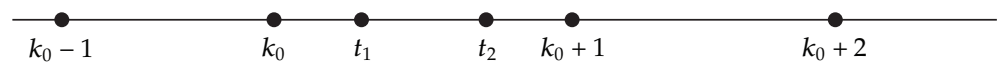

(b)

Figure 1: The relative position of $t_{1}$ and $t_{2}$.

Proof. Assume without loss of generality that $x(t)>0, L_{1} x(t)>0$ for $t \geq T$. We assert firstly that for any integer $k \geq T, L_{2} x(k) \geq 0$. If this is not true, then there exists an integer $j \geq T$ such that $L_{2} x(j):=\mu<0$. By (1.1), we obtain for $t \in[j+n, j+n+1), n=0,1,2, \ldots$, that

$$
\left(L_{2} x(t)\right)^{\prime}=-p(t) x^{\prime}(t)-f(t, x([t]))<0 .
$$

This implies that $L_{2} x(t) \leq L_{2} x(j)=\mu<0, t \in[j, j+1)$, and hence

$$
L_{2} x(j+1)=L_{2} x\left((j+1)^{-}\right) \leq \mu<0 .
$$

Using induction, we obtain that $L_{2} x(j+n) \leq L_{2} x(j)=\mu<0, n=0,1,2, \ldots$ Thus

$$
L_{2} x(t) \leq L_{2} x(j+n) \leq \mu<0, \quad t \in[j+n, j+n+1) .
$$

Integrating this inequality from $j+n$ to $t$, we find

$$
L_{1} x(t) \leq L_{1} x(j+n)+\mu R_{2}(t, j+n), \quad t \in[j+n, j+n+1) .
$$

Letting $t \rightarrow(j+n+1)^{-}$, it follows from (2.18) and the continuity of $L_{1} x(t)$ that

$$
L_{1} x(j+n+1) \leq L_{1} x(j+n)+\mu R_{2}(j+n+1, j+n) .
$$

Consequently,

$$
\begin{aligned}
L_{1} x(j+m+1) & =\sum_{n=0}^{m}\left(L_{1} x(j+n+1)-L_{1} x(j+n)\right)+L_{1} x(j) \\
& \leq \mu \sum_{n=0}^{m} R_{2}(j+n+1, j+n)+L_{1} x(j) \\
& =\mu R_{2}(j+m+1, j)+L_{1} x(j) \longrightarrow-\infty, \quad \text { as } m \longrightarrow \infty
\end{aligned}
$$

This contradicts that $L_{1} x(t)>0, t \geq T$. Therefore, $L_{2} x(k) \geq 0$ for any integer $k \geq T$. By the continuity and monotonicity of $L_{2} x(t)$, we get that $L_{2} x(t) \geq 0, t \geq T$. Finally, since $L_{2}^{\prime} x(t)>0$, we conclude that $L_{2} x(t)$ is eventually positive.

This completes the proof. 
Lemma 2.7. Let $x$ be a solution of (1.1) such that $x(t) x^{\prime}(t)<0, t \geq T$, where $T$ is a constant. If

$$
\lim _{t \rightarrow \infty} x(t)=\lambda \neq 0
$$

then for any arbitrary constant $\delta>1$, there exists an integer $k_{0} \geq T$ such that for any $t>s \geq k_{0}$, we have

$$
\begin{gathered}
\lambda<x(s)<\delta \lambda(\lambda>0) \quad \text { or } \quad \delta \lambda<x(s)<\lambda(\lambda<0) \\
(-1)^{x(t)}\left(L_{2} x(t)+p(t) x(t)-L_{2} x(s)-p(s) x(s)+\lambda \int_{s}^{t}\left[K q(u)-p^{\prime}(u)\right] d u\right) \geq 0 .
\end{gathered}
$$

Proof. Let us assume that $x(t)$ is eventually positive. The case when $x(t)$ is eventually negative can be similarly dealt with. For any constant $\delta>1$, it follows from (2.21) and $x \prime(t)<0$ that, there exists an integer $k_{0} \geq T$ such that $\lambda<x(t)<\delta \lambda$ for $t \geq k_{0}$. By (1.1), we have

$$
\left(L_{2} x(t)+p(t) x(t)\right)^{\prime}=p^{\prime}(t) x(t)-f(t, x([t])) \leq p^{\prime}(t) x(t)-q(t) \phi(x([t])), \quad t \in[k, k+1),
$$

where $k=k_{0}, k_{0}+1, k_{0}+2, \ldots$. Since $x([t])>x(t)$ and $\phi(x)$ is nondecreasing, it follows from (2.23) that

$$
\left(L_{2} x(t)+p(t) x(t)\right)^{\prime} \leq x(t)\left(p^{\prime}(t)-q(t) \frac{\phi(x([t]))}{x([t])}\right) \leq \lambda\left(p^{\prime}(t)-K q(t)\right), \quad t \in[k, k+1)
$$

Integrating (2.24) from $\xi$ to $\zeta$, where $k \leq \xi<\zeta<k+1$, we have

$$
L_{2} x(\zeta)+p(\zeta) x(\zeta) \leq L_{2} x(\xi)+p(\xi) x(\xi)+\lambda \int_{\xi}^{\zeta}\left(p^{\prime}(u)-K q(u)\right) d u .
$$

If there exists an integer $k_{1}$ such that $k_{1} \leq s<t<k_{1}+1$, then (2.22) follows from (2.25) directly. Otherwise, there exist an integer $k_{2}$ such that

$$
k_{2} \leq s<k_{2}+1<k_{2}+2<\cdots<k_{2}+m<t \leq k_{2}+m+1 .
$$


Using (2.25) and the continuity of $L_{2} x(t)$, we have that

$$
\begin{aligned}
& L_{2} x(t)+p(t) x(t)-L_{2} x(s)-p(s) x(s) \\
& =L_{2} x\left(k_{2}+1\right)+p\left(k_{2}+1\right) x\left(k_{2}+1\right)-L_{2} x(s)-p(s) x(s) \\
& +\sum_{n=1}^{m-1}\left[L_{2} x\left(k_{2}+n+1\right)+p\left(k_{2}+n+1\right) x\left(k_{2}+n+1\right)-L_{2} x\left(k_{2}+n\right)-p\left(k_{2}+n\right) x\left(k_{2}+n\right)\right] \\
& +L_{2} x(t)+p(t) x(t)-L_{2} x\left(k_{2}+m\right)-p\left(k_{2}+m\right) x\left(k_{2}+m\right) \\
& \leq \lambda \int_{s}^{k_{2}+1}\left(p^{\prime}(u)-K q(u)\right) d u+\lambda \sum_{n=1}^{m-1} \int_{k_{2}+n}^{k_{2}+n+1}\left(p^{\prime}(u)-K q(u)\right) d u \\
& +\lambda \int_{k_{2}+m}^{t}\left(p^{\prime}(u)-K q(u)\right) d u \\
& =\lambda \int_{s}^{t}\left(p^{\prime}(u)-K q(u)\right) d u \text {. }
\end{aligned}
$$

Thus we conclude that (2.22) holds for any $k_{0} \leq s<t$.

\section{Main Results}

In this section we present some sufficient conditions which guarantee that every solution of (1.1) oscillates or converges to zero. For convenience, we let

$$
\Phi(t)=\int_{t}^{\infty}\left[K q(\tau)-p^{\prime}(\tau)\right] d \tau, \quad t \geq 0
$$

Theorem 3.1. Suppose that

(i) (2.7) is nonoscillatory,

(ii) (2.5) and (2.6) are satisfied, and for every $T \geq 0$,

$$
\lim _{t \rightarrow \infty} \int_{T}^{t} q(s) \exp \left(\int_{T}^{s} \frac{p(u) R_{2}(u, T)}{r_{1}(u)} d u\right) d s=\infty,
$$

(iii) one of the following two conditions holds:

$$
\Phi(T)=\infty \text { for some } T \geq 0,
$$


or

$$
\begin{aligned}
& \text { for every } t \geq 0, \Phi(t)<\infty, \quad \text { and there exists a constant } \delta>1 \\
& \text { such that } \lim _{t \rightarrow \infty} \int_{T}^{t}\left(\frac{1}{r_{1}(s)} \int_{s}^{t} \frac{\Phi(u)-\delta p(u)}{r_{2}(u)} d u\right) d s=\infty .
\end{aligned}
$$

Then any solution $x$ of (1.1) is oscillatory or satisfies $x(t) \rightarrow 0$ as $t \rightarrow \infty$.

Proof. Let $x(t)$ be a nonoscillatory solution of (1.1). Without loss of generality, we may assume that $x(t)>0$ for $t \geq 0$. By Lemma 2.4, there exists a constant $T$ such that one of the following cases holds:

$$
\begin{array}{ll}
\text { Case } 1: x(t)>0, & x^{\prime}(t)>0, \quad t \geq T, \\
\text { Case 2: } x(t)>0, & x^{\prime}(t)<0, \quad t \geq T .
\end{array}
$$

We will firstly show that Case 1 is impossible. Indeed, if this case holds, then it follows from Lemma 2.6 that $x(t)$ has property $V_{2}$ on $\left[T_{1}, \infty\right)$ for some $T_{1} \geq T$. We define

$$
w(t)=\frac{L_{2} x(t)}{\phi(x([t]))}
$$

Clearly, $w(t)>0, t \geq T_{1}$. It follows from (1.1) that

$$
w^{\prime}(t)=-\frac{p(t) x^{\prime}(t)+f(t, x(k))}{\phi(x(k))} \leq-q(t)-p(t) \frac{L_{2} x(t)}{\phi(x(k))} \cdot \frac{x^{\prime}(t)}{L_{2} x(t)}, \quad t \in[k, k+1)
$$

where $k=\left[T_{1}\right]+1,\left[T_{1}\right]+2, \ldots$. Noting that

$$
L_{1} x(t)=L_{1} x\left(T_{1}\right)+\int_{T_{1}}^{t} \frac{L_{2} x(u)}{r_{2}(u)} d u \geq L_{2} x(t) R_{2}\left(t, T_{1}\right), \quad t \geq T_{1}
$$

we get from (3.8) that

$$
w^{\prime}(t) \leq-q(t)-p(t) \frac{R_{2}\left(t, T_{1}\right)}{r_{1}(t)} w(t), \quad t \in[k, k+1) .
$$

Denoted by

$$
\Psi(t)=w(t) \exp \left(\int_{T_{1}}^{t} p(s) \frac{R_{2}\left(s, T_{1}\right)}{r_{1}(s)} d s\right)
$$


then we get from (3.10) that

$$
\Psi^{\prime}(t) \leq-q(t) \exp \left(\int_{T_{1}}^{t} p(s) \frac{R_{2}\left(s, T_{1}\right)}{r_{1}(s)} d s\right), \quad t \in[k, k+1)
$$

Integrating (3.12), we have

$$
\Psi\left((k+1)^{-}\right)-\Psi(k) \leq-\int_{k}^{k+1} q(t) \exp \left(\int_{T_{1}}^{t} p(s) \frac{R_{2}\left(s, T_{1}\right)}{r_{1}(s)} d s\right) d t
$$

Using the increasing property of $x(t)$, it follows that

$$
\begin{aligned}
\Psi(k+1) & =\frac{L_{2} x(k+1)}{\phi(x(k+1))} \exp \left(\int_{T_{1}}^{k+1} p(s) \frac{R_{2}\left(s, T_{1}\right)}{r_{1}(s)} d s\right) \\
& \leq \frac{L_{2} x(k+1)}{\phi(x(k))} \exp \left(\int_{T_{1}}^{k+1} p(s) \frac{R_{2}\left(s, T_{1}\right)}{r_{1}(s)} d s\right)=\Psi\left((k+1)^{-}\right) .
\end{aligned}
$$

Thus

$$
\begin{aligned}
\Psi(k+m) & =\Psi(k)+\sum_{n=k+1}^{n=k+m}[\Psi(n)-\Psi(n-1)] \\
& \leq \Psi(k)+\sum_{n=k+1}^{n=k+m}\left[\Psi\left(n^{-}\right)-\Psi(n-1)\right] \\
& \leq \Psi(k)-\int_{k}^{k+m} q(t) \exp \left(\int_{T_{1}}^{t} p(s) \frac{R_{2}\left(s, T_{1}\right)}{r_{1}(s)} d s\right) d t
\end{aligned}
$$

where $m=1,2, \ldots$. For fixed $k$, by letting $m \rightarrow \infty$, we get from (3.2) that $\Psi(j)<0$ for large integer $j$, which is contrary to the fact that $\Psi(t)>0, t \geq T_{1}$.

Therefore, we only need to consider Case 2: $x(t)>0, x^{\prime}(t)<0, t \geq T$. We assert that

$$
\lim _{t \rightarrow \infty} x(t)=\lambda=0
$$

Suppose to the contrary that $\lambda>0$. For any arbitrary constant $\delta>1$, it follows from Lemma 2.7 that, there exists an integer $k_{0} \geq T$ such that $\lambda<x(t)<\delta \lambda\left(t \geq k_{0}\right)$ and

$$
L_{2} x(t)+p(t) x(t) \leq L_{2} x(s)+p(s) x(s)-\lambda \int_{s}^{t}\left[K q(u)-p^{\prime}(u)\right] d u
$$

where $t>s \geq k_{0}$. 
Now assume that (3.3) holds. Letting $s=k_{0}$ in (3.17), we have

$$
L_{2} x(t) \leq M-\lambda \int_{k_{0}}^{t}\left[K q(u)-p^{\prime}(u)\right] d u,
$$

where $M$ is a constant. By this inequality and (3.3), there exists a negative constant $\mu$ and a positive constant $T_{1} \geq k_{0}$ such that $L_{2} x(t)<\mu$ for $t \geq T_{1}$. Integrating $\left(r_{1}(t) x \prime(t)\right) \prime<\mu r_{2}^{-1}(t)$ from $T_{1}$ to $t$ twice, we have

$$
x(t) \leq x\left(T_{1}\right)+L_{1} x\left(T_{1}\right) R_{1}\left(t, T_{1}\right)+\mu \int_{T_{1}}^{t} \frac{R_{2}\left(s, T_{1}\right)}{r_{1}(s)} d s .
$$

Thus we get from (2.5) that $x(t)<0$ for large $t$, a contradiction.

Next, assume that (3.4) holds. We distinguish the following three subcases: (i) $L_{2} x(t) \leq$ 0 for all large $t$, (ii) $L_{2} x(t) \geq 0$ for all large $t$, (iii) $L_{2} x(t)$ changes sign for arbitrarily large $t$.

Case (i) is equivalent to that $x^{\prime \prime}(t) \leq 0$ for all large $t$. Since $x^{\prime}(t)<0$, we conclude that $x(t)$ is eventually negative, a contradiction.

In case (ii), we get from (2.22) that

$$
L_{2} x(s)+p(s) x(s) \geq \lambda \int_{s}^{t}\left[K q(u)-p^{\prime}(u)\right] d u, \quad t>s \geq T_{2} \geq k_{0}
$$

where $T_{2}$ is a constant such that $L_{2} x(t) \geq 0$ on $\left[T_{2}, \infty\right)$. Letting $t \rightarrow \infty$, we obtain that

$$
L_{2} x(s)+\delta \lambda p(s) \geq \lambda \Phi(s)
$$

Integrating (3.21) from $s$ to $t$, using $L_{1} x(t) \leq 0$, it turns out that

$$
L_{1} x(s) \leq \delta \lambda \int_{s}^{t} \frac{p(u)}{r_{2}(u)} d u-\lambda \int_{s}^{t} \frac{\Phi(u)}{r_{2}(u)} d u
$$

Integrating again from $T_{2}$ to $t$, we find

$$
x(t) \leq x\left(T_{2}\right)+\int_{T_{2}}^{t}\left(\frac{\delta \lambda}{r_{1}(s)} \int_{s}^{t} \frac{p(u)}{r_{2}(u)} d u\right) d s-\int_{T_{2}}^{t}\left(\frac{\lambda}{r_{1}(s)} \int_{s}^{t} \frac{\Phi(u)}{r_{2}(u)} \mathrm{d} u\right) d s .
$$

By the condition (3.4), we have that $x(t)<0$ for all large $t$. This is a contradiction.

Finally, in case (iii), we let $\left\{t_{n}\right\}$ be the sequence of zeros of $L_{2} x(t)$ such that $\lim _{n \rightarrow \infty} t_{n}=$ $\infty$. By choosing $t=t_{n}$ in (2.22), we get

$$
L_{2} x(s)+p(s) x(s) \geq \lambda \int_{s}^{t_{n}}\left[K q(u)-p^{\prime}(u)\right] d u .
$$


Let $n \rightarrow \infty$, it follows that

$$
L_{2} x(s)+\delta \lambda p(s) \geq \lambda \Phi(s)
$$

Integrating this inequality twice yields that

$$
x\left(t_{n}\right) \leq x\left(T_{0}\right)+\int_{T_{0}}^{t_{n}}\left(\frac{\delta \lambda}{r_{1}(s)} \int_{s}^{t_{n}} \frac{p(u)}{r_{2}(u)} d u\right) d s-\int_{T_{0}}^{t_{n}}\left(\frac{\lambda}{r_{1}(s)} \int_{s}^{t_{n}} \frac{\Phi(u)}{r_{2}(u)} d u\right) d s,
$$

which also leads to a contradiction.

Therefore, in Case 2 we conclude that (3.16) holds. This completes the proof of the theorem.

From the conclusion of Theorem 3.1, we get the following corollary.

Corollary 3.2. Suppose that (2.5), (2.6) hold and that (2.7) is nonoscillatory. Assume further that $p^{\prime}(t) \leq 0$ for large t. If $\int_{T}^{\infty} q(t) d t=\infty$, then any solution $x$ of (1.1) is oscillatory or satisfies $x(t) \rightarrow 0$ as $t \rightarrow \infty$.

Proof. Since $p^{\prime}(t) \leq 0$, it follows that

$$
\Phi(T)=\int_{T}^{\infty}\left[K q(\tau)-p^{\prime}(\tau)\right] d \tau \geq K \int_{T}^{\infty} q(s) d s=\infty
$$

Thus (3.3) is satisfied.

On the other hand, with the condition $\int_{T}^{\infty} q(t) d t=\infty$ we get (3.2) immediately. Consequently, the expected conclusion follows from Theorem 3.1 directly.

We next consider the following equation which is different from (1.1):

$$
\left(r_{2}(t)\left(r_{1}(t) x^{\prime}(t)\right)^{\prime}\right)^{\prime}+p(t) x^{\prime}(t)+q(t) x^{\alpha}([t])=0, \quad t \geq 0,
$$

or the more general equation

$$
\left(r_{2}(t)\left(r_{1}(t) x^{\prime}(t)\right)^{\prime}\right)^{\prime}+p(t) x^{\prime}(t)+\bar{f}(t, x([t]))=0, \quad t \geq 0,
$$

with

$$
|\bar{f}(t, x)| \geq q(t)|\bar{\phi}(x)|, \quad \frac{\bar{\phi}(x)}{x^{\alpha}} \geq K>0, \quad q(t)>0,
$$

where $\alpha \in(0,1) \cup(1, \infty)$ is a quotient of odd integers. We note that the result of Theorem 3.1 is not applicable. In what follows we give an oscillation criteria for (3.29). 
Let

$$
\Phi_{1}(t)=\int_{t}^{\infty}\left[K_{1} q(\tau)-p^{\prime}(\tau)\right] d \tau, \quad t \geq 0
$$

Suppose that $K_{1} q(t)-p^{\prime}(t) \geq 0$ and not identically zero in any subinterval of $[0, \infty)$ for any $K_{1}>0$. Moreover, we need the following conditions:

$$
\Phi_{1}(T)=\infty, \quad \text { for some } T \geq 0,
$$

or

$$
\begin{aligned}
& \text { for every } t \geq 0, \Phi_{1}(t)<\infty, \quad \text { and there exists a } \delta>1, \\
& \text { such that } \lim _{t \rightarrow \infty} \int_{T}^{t}\left(\frac{1}{r_{1}(s)} \int_{s}^{t} \frac{\Phi_{1}(u)-\delta p(u)}{r_{2}(u)} d u\right) d s=\infty
\end{aligned}
$$

Theorem 3.3. Suppose that

(i) (2.7) is nonoscillatory,

(ii) (2.5) and (2.6) are satisfied, and for every $T \geq 0$,

$$
\lim _{t \rightarrow \infty} \int_{T}^{t} q(s) \exp \left(\int_{T}^{s} \frac{p(u) R_{2}(u, T)}{r_{1}(u)} d u\right) d s=\infty
$$

(iii) for any $K_{1}>0$, one of (3.32) and (3.33) is satisfied.

Then any solution $x$ of (3.29) is oscillatory or satisfies $x(t) \rightarrow 0$ as $t \rightarrow \infty$.

Proof. From the proof of Theorem 3.1, it suffices to verify in Case 2 that there exists a positive constant $K_{1}$ such that

$$
L_{2} x(t)+p(t) x(t) \leq L_{2} x(s)+p(s) x(s)-\lambda \int_{s}^{t}\left[K_{1} q(u)-p^{\prime}(u)\right] d u,
$$

for any $t>s \geq k_{0}$, where $k_{0} \geq T$ is the integer such that $\lambda<x(t)<\delta \lambda, t \geq k_{0}$.

By (3.29), we obtain that

$$
\begin{aligned}
\left(L_{2} x(t)+p(t) x(t)\right)^{\prime} & =p^{\prime}(t) x(t)-\bar{f}(t, x([t])) \\
& \leq x(t)\left(p^{\prime}(t)-q(t) \frac{\bar{\phi}(x([t]))}{x([t])}\right) \\
& \leq x(t)\left(p^{\prime}(t)-K q(t) x^{\alpha-1}([t])\right),
\end{aligned}
$$


$t \in[k, k+1)$, where $k=k_{0}, k_{0}+1, k_{0}+2, \ldots$. If $\alpha>1$, then it follows from this inequality that

$$
\left(L_{2} x(t)+p(t) x(t)\right)^{\prime} \leq \lambda\left(p^{\prime}(t)-K \lambda^{\alpha-1} q(t)\right)
$$

while $\alpha<1$, we have

$$
\left(L_{2} x(t)+p(t) x(t)\right)^{\prime} \leq \lambda\left(p^{\prime}(t)-K(\delta \lambda)^{\alpha-1} q(t)\right),
$$

Let $K_{1}=K \lambda^{\alpha-1}$ if $\alpha>1$ and $K_{1}=K(\delta \lambda)^{\alpha-1}$ if $\alpha<1$, it turns out for the both cases that

$$
\left(L_{2} x(t)+p(t) x(t)\right)^{\prime} \leq \lambda\left(p^{\prime}(t)-K_{1} q(t)\right), \quad t \in[k, k+1) .
$$

The rest of the proof is exactly the same as in Theorem 3.1 and hence is omitted.

The following result follows from Theorem 3.3 directly.

Corollary 3.4. Suppose that (2.5), (2.6) hold and that (2.7) is nonoscillatory. Assume further that $p^{\prime}(t) \leq 0$ for large $t$. If $\int_{T}^{\infty} q(t) d t=\infty$, then any solution $x(t)$ of (3.29) is oscillatory or satisfies $x(t) \rightarrow 0$ as $t \rightarrow \infty$.

Remark 3.5. In the literature dealing with the third-order delay differential equation, the Riccati transformation $w(t)=\rho(t) L_{2} x(t) / x(g(t))$, where $g(t)$ is the delay and $\rho(t)$ is a differentiable positive function, is used widely, see $[3,4]$ and the references therein. However, in our paper we find that the transformation $w(t)=L_{2} x(t) / \phi(x([t]))$ (see (3.7)) plays the same role as the more general one

$$
w(t)=\frac{\rho(t) L_{2} x(t)}{\phi(x([t]))} .
$$

In fact, if we replace (3.7) by (3.40) in the proof of Theorem 3.1, then it yields that

$$
w^{\prime}(t) \leq-\rho(t) q(t)+\left(\frac{\rho^{\prime}(t)}{\rho(t)}-p(t) \frac{R_{2}(t, T)}{r_{1}(t)}\right) w(t) .
$$

Similarly to Theorem 3.1, we need the following condition (instead of (3.2))

$$
\lim _{t \rightarrow \infty} \int_{T}^{t} \rho(s) q(s) \exp \left(\int_{T}^{s}-\frac{\rho^{\prime}(u)}{\rho(u)}+p(u) \frac{\mathrm{R}_{2}(u, T)}{r_{1}(t)} d u\right) d s=\infty
$$

to obtain a contradiction. Noting that

$$
\rho(s) \exp \left(-\int_{T}^{s} \frac{\rho^{\prime}(u)}{\rho(u)} d u\right)=\rho(s) \exp \left(-\int_{\rho(T)}^{\rho(s)} \frac{d \rho}{\rho}\right)=\rho(T),
$$

we conclude that (3.42) is equivalent to (3.2). 
Therefore, we cannot get a more general result by using the "more general" transformation (3.40). This is different from the theory of function differential equation with the continuous delay.

\section{Examples}

In this section, we give several examples to illustrate our main results. For the convenience of readers, let us firstly recall the famous lemma of Kneser [13]. Consider the following secondorder ordinary differential equation:

$$
y^{\prime \prime}(t)+a(t) y(t)=0
$$

where $a(t)$ is a locally integrable function of $t$. Kneser [13] shows that (4.1) is nonoscillatory if $a(t) \leq\left(1 / 4 t^{2}\right)$ and is oscillatory if $a(t) \geq(1+\varepsilon) / 4 t^{2}$, where $\varepsilon$ is an any arbitrary positive constant.

Example 4.1. Consider the third-order delay differential equation with piecewise constant argument

$$
x^{\prime \prime \prime}(t)+\frac{3 \sqrt{t}}{e^{t}+17 t^{2}} x^{\prime}(t)+\left(1+t^{2}\right) x^{\alpha}([t]) e^{1+t x([t])}=0, \quad t \geq 0,
$$

where $\alpha>0$ is a quotient of odd integers. Clearly, $p(t)=3 \sqrt{t} /\left(e^{t}+17 t^{2}\right), f(t, x)=t^{2} x^{\alpha} e^{1+t x}$. Let $q(t)=1+t^{2}, \phi(x)=x^{\alpha}$, then $|f(t, x)| \geq q(t)|\phi(x)|$. By the results of [13], $y^{\prime \prime}(t)+p(t) y(t)=0$ is nonoscillatory. A simple calculation shows that $p \prime(t)<0$ for $t>1$. Therefore, it is easy to see from Corollary 3.4 that any solution of (4.2) is either oscillatory or converges to zero.

Example 4.2. Consider the third-order delay differential equation with piecewise constant argument

$$
\left((t+1) x^{\prime}(t)\right)^{\prime \prime}+\frac{1}{5 t+5} x^{\prime}(t)+(t+1)^{-6 / 5} x([t])\left(1+e^{x([t])}\right)=0, \quad t \geq 0
$$

where $r_{1}(t)=t+1, r_{2}(t)=1, p(t)=(5 t+5)^{-1}$, and $f(t, x)=(t+1)^{-6 / 5} x\left(1+e^{x}\right)$. Let $q(t)=(t+$ $1)^{-6 / 5}, \phi(x)=x$, then $|f(t, x)| \geq q(t)|\phi(x)|$. It is easy to see that the Euler equation $5 \tau^{2} z^{\prime \prime}(\tau)+$ $z(\tau)=0$ is nonoscillatory, hence $5(t+1)^{2} y^{\prime \prime}(t)+y(t)=0$ is nonoscillatory. A simple calculation leads to

$$
\begin{gathered}
\Phi(u)=\int_{u}^{\infty}\left[q(\tau)-p^{\prime}(\tau)\right] d \tau=\frac{5}{(u+1)^{1 / 5}}+\frac{1}{5 u+5^{\prime}} \\
\Phi(u)-2 p(u) \geq \frac{4}{5(u+1)^{1 / 5}}, \quad \text { and hence } \int_{s}^{t} \frac{\Phi(u)-2 p(u)}{r_{2}(u)} d u \geq(1+t)^{4 / 5}-(1+s)^{4 / 5} .
\end{gathered}
$$


Thus

$$
\int_{T}^{t} \frac{1}{r_{1}(s)} \int_{s}^{t} \frac{\Phi(u)-2 p(u)}{r_{2}(u)} d u d s \geq\left[\ln (1+t)-\frac{5}{4}-\ln (1+T)\right](1+t)^{4 / 5}+\frac{5}{4}(1+T)^{4 / 5} \longrightarrow \infty,
$$

as $t \rightarrow \infty$.

On the other hand,

$$
\exp \left(\int_{T}^{t} \frac{p(u) R_{2}(u, T)}{r_{1}(u)} d u\right)=\frac{1}{5} \int_{T}^{t} \frac{(s-T)}{(s+1)^{2}} d s=\frac{1}{5}\left[\frac{1+T}{1+t}+\ln (1+t)-1-\ln (1+T)\right]
$$

which yields that

$$
\exp \left(\int_{T}^{t} \frac{p(u) R_{2}(u, T)}{r_{1}(u)} d u\right)=a e^{b(1+t)^{-1}}(1+t)^{1 / 5} \geq a(1+t)^{1 / 5}
$$

where $a, b$ are positive constants. Hence

$$
\int_{T}^{t} q(s) \exp \left(\int_{T}^{s} \frac{p(u) R_{2}(u, T)}{r_{1}(u)} d u\right) d s \geq a \int_{T}^{t} \frac{1}{1+s} d s \longrightarrow \infty \quad \text { as } t \longrightarrow \infty
$$

Obviously, the other conditions of Theorem 3.1 are also satisfied. Hence we conclude that any solution of (4.3) is either oscillatory or converges to zero.

Example 4.3. Consider the third-order delay differential equation with piecewise constant argument

$$
\left(e^{-t}\left(e^{-t} x^{\prime}\right)^{\prime}\right)^{\prime}+\frac{e^{-2 t}}{4} x^{\prime}(t)+\frac{3 t}{2+\sin 2 t} \ln \left(\frac{t+1}{\sqrt{2}}\right) x^{3}([t])=0, \quad t \geq 0
$$

Here $p(t)=e^{-2 t} / 4, f(t, x)=(3 t /(2+\sin 2 t)) \ln ((t+1) / \sqrt{2}) x^{3}$. Let $q(t)=(3 t /(2+\sin 2 t)) \ln ((t+$ $1) / \sqrt{2}), \phi(x)=x^{3}$, then $|f(t, x)|=q(t)|\phi(x)|$. Since the equation $e^{-t}\left(e^{-t} y^{\prime}\right)^{\prime}+p(t) y^{\prime}(t)=0$ can be reduced to $y^{\prime \prime}-y^{\prime}+(1 / 4) y=0$, we conclude that the former is nonoscillatory. It is easy to see that $p^{\prime}(t)<0$ and $\int_{0}^{\infty} q(t) d t=\infty$. Therefore, By Corollary 3.4, any solution of (4.9) is either oscillatory or converges to zero.

Example 4.4. Consider the third-order delay differential equation with piecewise constant argument

$$
\left(\frac{1}{\ln (1+t)}\left((2 t+3) x^{\prime}\right)^{\prime}\right)^{\prime}+\frac{1}{t^{2}(2+t)^{2}} x^{\prime}(t)+\left(g(t)-\frac{4(1+t)}{t^{3}(2+t)^{3}}\right) x^{\alpha}([t])=0, \quad t \geq 0
$$


where $\alpha>0$ is a quotient of odd integers $g(t)$ is a integrable function such that

$$
\int^{\infty} g(t) d t=\infty, \quad g(t)>\frac{4(1+t)}{t^{3}(2+t)^{3}} \quad(t>0)
$$

Let $r_{1}(t)=2 t+3, r_{2}(t)=1 / \ln (1+t), p(t)=1 / t^{2}(2+t)^{2}$, and $f(t, x)=\left(g(t)-4(1+t) / t^{3}(2+t)^{3}\right) x^{\alpha}$. We will show that

$$
\left(r_{2}(t) y^{\prime}(t)\right)^{\prime}+\frac{p(t)}{r_{1}(t)} y(t)=0
$$

is nonoscillatory. We introduce the change of variables:

$$
s=s(t)=\left[\int_{0}^{t} \ln (1+\tau) d \tau\right]^{-1}, \quad y(t)=s^{-1} z(s),
$$

which transforms (4.12) into

$$
z^{\prime \prime}(s)+\left(\left.\frac{p(t) r_{2}(t)}{s^{4} r_{1}(t)}\right|_{t=t(s)}\right) z(s)=0
$$

Note that

$$
\left.\frac{p(t) r_{2}(t)}{s^{4} r_{1}(t)}\right|_{s=s(t)}=\frac{\left(\int_{0}^{t} \ln (1+\tau) d \tau\right)^{4}}{(2 t+3) t^{2}(2+t)^{2} \ln (1+t)},
$$

and that

$$
\begin{gathered}
t^{2}(2+t)^{2}=4\left(\frac{t^{2}}{2}+t\right)^{2}=4\left(\int_{0}^{t}(1+\tau) d \tau\right)^{2} \geq 4\left(\int_{0}^{t} \ln (1+\tau) d \tau\right)^{2} \\
(2 t+3) \ln (1+t)>1, \quad t \geq 2,
\end{gathered}
$$

we obtain that

$$
\left.\frac{p(t) r_{2}(t)}{s^{4} r_{1}(t)}\right|_{s=s(t)} \leq \frac{\left(\int_{0}^{t} \ln (1+\tau) d \tau\right)^{4}}{4\left(\int_{0}^{t} \ln (1+\tau) d \tau\right)^{2}}=\frac{1}{4 s^{2}}
$$

Thus by Kneser [13], it follows that (4.14) (and hence (4.12)) is nonoscillatory.

Let $q(t)=g(t)-4(1+t) / t^{3}(2+t)^{3}$, then it is easy to find that $\int^{\infty} q(t) d t=\infty$. Therefore, by Corollary 3.4, any solution of (4.10) is either oscillatory or converges to zero. 


\section{Acknowledgments}

The authors would like to thank the referee very much for his valuable comments and suggestions. Liang was supported by the NSF of China (no. 11201086) and Foundation for Distinguished Young Talents in Higher Education of Guangdong, China (Grant 2012LYM_0087).

\section{References}

[1] R. P. Agarwal, S. R. Grace, and D. O'Regan, Oscillation Theory for Difference and Functional Differential Equations, Kluwer Academic, Dordrecht, The Netherlands, 2000.

[2] O. Došlý and A. Lomtatidze, "Oscillation and nonoscillation criteria for half-linear second order differential equations," Hiroshima Mathematical Journal, vol. 36, no. 2, pp. 203-219, 2006.

[3] S. H. Saker, "Oscillation criteria of third-order nonlinear delay differential equations," Mathematica Slovaca, vol. 56, no. 4, pp. 433-450, 2006.

[4] A. Tiryaki and M. F. Aktaş, "Oscillation criteria of a certain class of third order nonlinear delay differential equations with damping," Journal of Mathematical Analysis and Applications, vol. 325, no. 1, pp. 54-68, 2007.

[5] K. L. Cooke and J. Wiener, "A survey of differential equations with piecewise continuous arguments," in Delay Differential Equations and Dynamical Systems, vol. 1475 of Lecture Notes in Mathematics, pp. 1-15, Springer, Berlin, Germany, 1991.

[6] A. R. Aftabizadeh and J. Wiener, "Oscillatory properties of first order linear functional-differential equations," Applicable Analysis, vol. 20, no. 3-4, pp. 165-187, 1985.

[7] A. R. Aftabizadeh, J. Wiener, and J.-M. Xu, "Oscillatory and periodic solutions of delay differential equations with piecewise constant argument," Proceedings of the American Mathematical Society, vol. 99, no. 4, pp. 673-679, 1987.

[8] H. A. Agwo, "Necessary and sufficient conditions for the oscillation of delay differential equation with a piecewise constant argument," International Journal of Mathematics and Mathematical Sciences, vol. 21, no. 3, pp. 493-497, 1998.

[9] Z. Luo and J. Shen, "New results on oscillation for delay differential equations with piecewise constant argument," Computers \& Mathematics with Applications, vol. 45, no. 12, pp. 1841-1848, 2003.

[10] G.-Q. Wang and S. S. Cheng, "Oscillation of second order differential equation with piecewise constant argument," Cubo, vol. 6, no. 3, pp. 55-63, 2004.

[11] S. M. Shan and J. Wiener, "Advanced differential equations with piecewise constant argument deviations," International Journal of Mathematics and Mathematical Sciences, vol. 6, pp. 55-63, 1983.

[12] A. Škerlík, "Oscillation theorems for third order nonlinear differential equations," Mathematica Slovaca, vol. 42, no. 4, pp. 471-484, 1992.

[13] A. Kneser, "Untersuchungen über die reellen Nullstellen der Integrale linearer Differentialgleichungen," Mathematische Annalen, vol. 42, no. 3, pp. 409-435, 1893. 


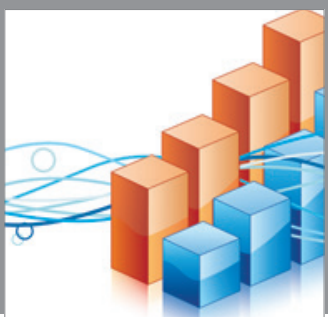

Advances in

Operations Research

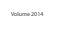

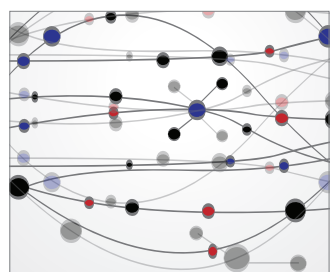

\section{The Scientific} World Journal
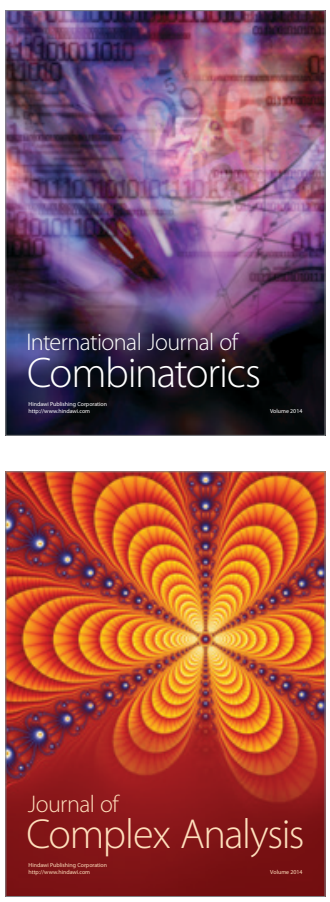

International Journal of

Mathematics and

Mathematical

Sciences
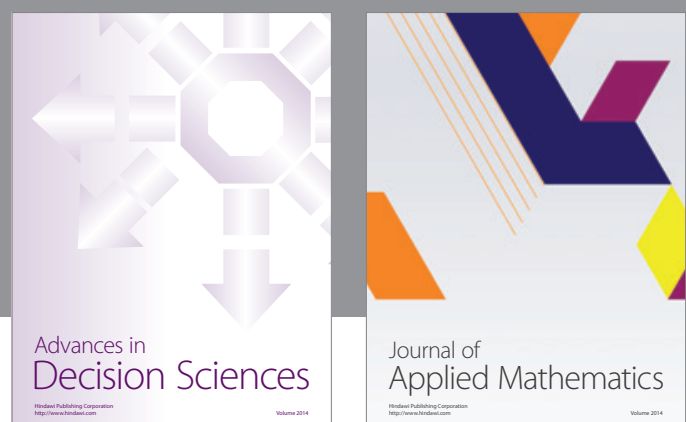

Journal of

Applied Mathematics
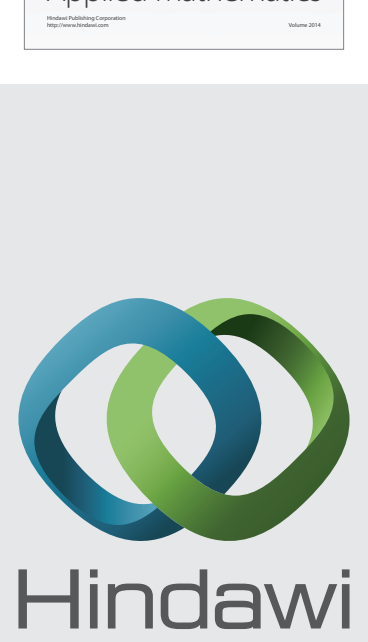

Submit your manuscripts at http://www.hindawi.com
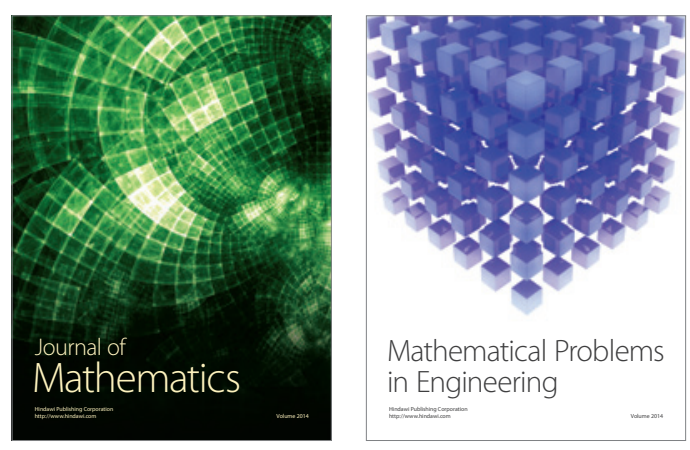

Mathematical Problems in Engineering
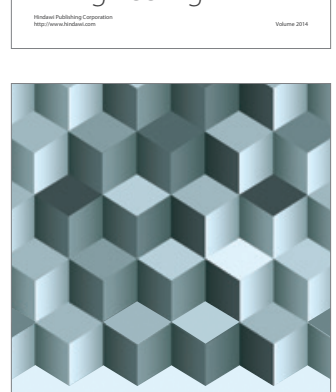

Journal of

Function Spaces
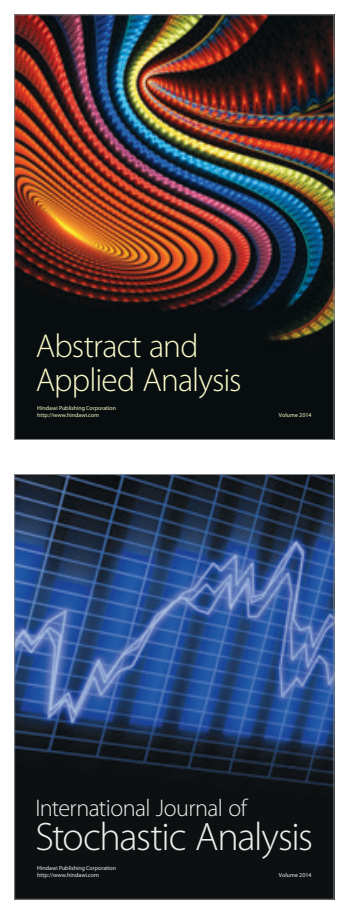

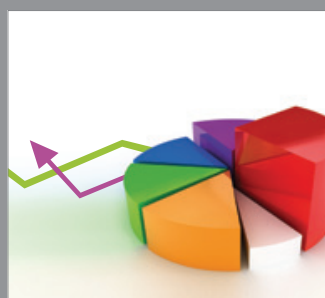

ournal of

Probability and Statistics

Promensencen
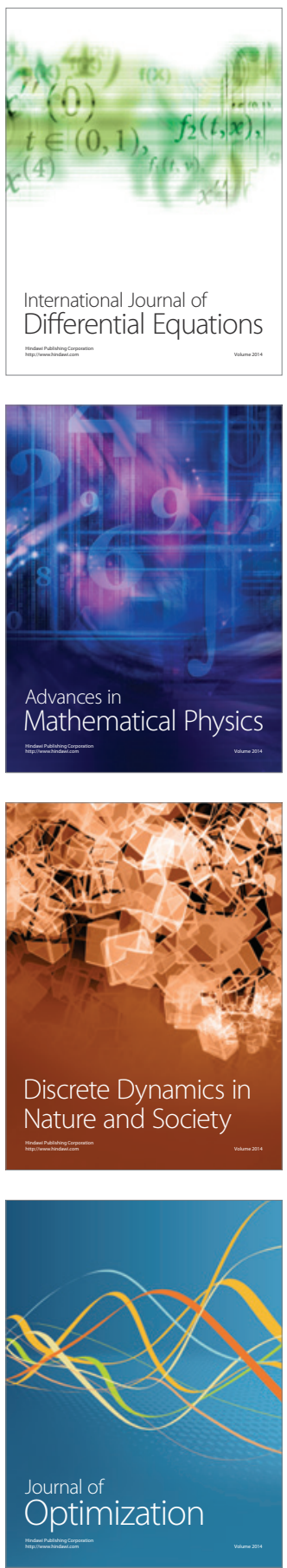